\title{
Smoking and antidepressants pharmacokinetics: a systematic review
}

\author{
Pedro Oliveira ${ }^{1}$, Joana Ribeiro ${ }^{1}$, Helena Donato ${ }^{2}$ and Nuno Madeira ${ }^{1 *}$ (D)
}

\begin{abstract}
Background: Despite an increasingly recognized relationship between depression and smoking, little is known about how smoking influences antidepressant response and treatment outcomes. The aim of this study was to systematically review the evidence of the impact of smoking on new-generation antidepressants with an emphasis on the pharmacokinetic perspective.
\end{abstract}

Methods: We present a systematic review of clinical trials comparing the serum levels of new-generation antidepressants in smokers and nonsmokers. Data were obtained from MEDLINE/PubMed, Embase, and other sources. Risk of bias was assessed for selection, performance, detection, attrition, and reporting of individual studies.

Results: Twenty-one studies met inclusion criteria; seven involved fluvoxamine, two evaluated fluoxetine, sertraline, venlafaxine, duloxetine or mirtazapine, and escitalopram, citalopram, trazodone and bupropion were the subject of a single study. No trials were found involving other common antidepressants such as paroxetine or agomelatine. Serum levels of fluvoxamine, duloxetine, mirtazapine and trazodone were significantly higher in nonsmokers compared with smokers.

Conclusions: There is evidence showing a reduction in the concentration of serum levels of fluvoxamine, duloxetine, mirtazapine and trazodone in smoking patients as compared to nonsmokers. The evidence regarding other commonly used antidepressants is scarce. Nonetheless, smoking status should be considered when choosing an antidepressant treatment, given the risk of pharmacokinetic interactions.

Keywords: Antidepressant agents, Pharmacokinetics, Depressive disorder, Smoking

\section{Background}

One-quarter of the general population, $40-50 \%$ of people with depression and $70-80 \%$ of those with schizophrenia smoke $[1,2]$. Major depressive disorder (MDD) is a common psychiatric disease and a major public health problem. It has been projected to become the leading cause of disability and the second leading contributor to the global burden of disease and overall mortality by the year 2020; according to the recent epidemiologic data, about $10 \%$ of world population suffers with depression [3]. The advent of antidepressants (AD), starting with imipramine in 1958, has revolutionized the treatment of depression;

\footnotetext{
${ }^{*}$ Correspondence: nunogmadeira@gmail.com

1 Psychiatry Department, Coimbra Hospital University Centre, Praceta Mota Pinto, 3000-075 Coimbra, Portugal

Full list of author information is available at the end of the article
}

yet this first generation of AD had an unfavorable adverse effect profile that has improved significantly with the advent in the late 1980s of selective serotonin reuptake inhibitors (SSRI) and, some years later, of serotonin and noradrenaline reuptake inhibitors (SNRI) and others like mirtazapine, trazodone, agomelatine and bupropion [4]. Given their favorable adverse effects profile, new-generation antidepressants are the first-line treatment of MDD. The mechanism of action and efficacy is similar between drugs of the same pharmacological class. However, there are considerable differences among interindividual responses to a given drug. Many theories tried to explain this variation but the most accepted is that clinical response is related to serum levels of the antidepressants. For many SSRI and other new-generation antidepressant drugs, a relationship between plasma concentrations and clinical effects is not reported [5]. However, serum levels 
below therapeutic range could compromise the clinical response [5]. The serum levels of antidepressants are essentially dependent on two variables: the intake dose of drug and the rate of elimination. The rate of elimination of antidepressants is almost exclusively dependent on their hepatic metabolization. The hepatic metabolism of AD is made by cytochrome P450 (CYP) which is a group of many enzymes that exists in different amounts in human liver. Different antidepressants are metabolized by different subtypes of CYP and the amount of each CYP varies from person to person [6]. Thus, it is now accepted that the difference found in the response to an antidepressant could be correlated with serum concentrations. With the exception of tricyclic antidepressants, the correlation between plasmatic levels and the clinical outcome is still not consensual [7]. However, AGNP Consensus Guidelines from 2011 attribute a level 2 of recommendation for therapeutic drug monitoring for SSRI (except paroxetine) and SNRI [7]. Despite the availability of techniques capable of quantifying the activity of CYP isozymes such as $1 \mathrm{~A} 2,2 \mathrm{~B} 6,2 \mathrm{C} 9$ and $3 \mathrm{~A} 4$ [8], they are not commonly used in clinical practice. So, given the absence of clinical indicators as relating to the higher or lower individual activity of each CYP, often the choice is made based on clinical experience with the particular drug and previous response of the individual to antidepressants in the past. Such imprecision can lead to the inappropriate use of drugs, not only wasting time until an adequate remission of depression but also causing iatrogenic harm, jeopardizing patient trust in antidepressant treatments.

There are 4000 chemical compounds found in cigarette smoke and 43 have been identified to be carcinogenic. Cigarette smoke constituents have been shown to stimulate or induce hepatic CYP isozymes, which play a central role in drug metabolism. Polycyclic aromatic hydrocarbons (PAH) from cigarette smoke are responsible for the induction of CYP isozymes. PAHs have been shown to induce CYP1A1, CYP1A2 and CYP2E1 [9].

Given the possibility that some antidepressants are metabolized by CYP induced or inhibited by substances in tobacco, their identification can be a guide for drug initial choice in smoking patients, allowing a more accurate antidepressant selection and consequently improving the pharmacologic treatment of depression in smokers.

Cigarette smoking can affect the clinical management of patients with psychiatric disorders because of the pharmacokinetic and pharmacodynamical changes; it can cause to various psychotropic drugs. This article reviews the impact of smoking on new-generation antidepressants with an emphasis on the pharmacokinetic perspective. It also seeks to provide critical information on whether such variations should influence antidepressant choice in this population.

\section{Methods}

This review was performed according to the PRISMA guidelines [10], thus providing a comprehensive framework which objectively assesses indicators of quality and risk of biases of included studies.

All original studies investigating the difference between the levels of any new-generation antidepressive agents (SSRI, SNRI, trazodone, mirtazapine, bupropion or agomelatine) and the smoking status were eligible for this systematic review. Further criteria adopted were: (1) publication date between January 1970 and June 2016, (2) empirical study, (3) written in English or Portuguese language, (4) published in a scholarly peer-reviewed journal, (5) studies that determined serum levels within a steady state, and (6) comparison of serum levels between 2 groups-smokers and nonsmokers. Additionally, studies were excluded from review if they were: (1) single-case report, (2) review articles, (3) repeated study population, (4) comparisons involving combinations of drugs, (5) animal studies, and (6) trials involving only metabolites.

Studies were identified by searching relevant papers via PubMed/MEDLINE (http://www.ncbi.nlm.nih.gov/ pubmed), Cochrane Library and EMBASE using the following keywords: ("antidepressive agents") AND (smok"). Finally, reference lists of retrieved studies were hand searched to identify any additional relevant studies. Keywords and combination of keywords were used to search the electronic databases and were organized following the population intervention comparison outcome (PICO) model (Fig. 1). In this model, the search strategy can be organized based on the topics: population $(\mathrm{P})$, intervention $(\mathrm{I})$, control group $(\mathrm{C})$, and outcome $(\mathrm{O})$ and several searches in the aforementioned databases.

After performing the initial literature searches, each study title and abstract was screened for eligibility by the first author. Full text of all potentially relevant studies were subsequently retrieved and further examined for eligibility. The PRISMA flow diagram (Fig. 1) provides more detailed information regarding the selection process of studies. Information from the included studies was then analyzed and recorded in an electronic spreadsheet designed by the first author. Different types of data were extracted from each study including: (a) country in which the data were collected and participants' characteristics, (b) number of subjects, (c) number of smokers, (d) age average, (e) percentage of males, (f) main results (g) intervention protocol, (h) risk of bias in individual studies, and (i) limitations among others. The Cochrane Collaboration's tool for assessing risk of bias was adopted to evaluate the risk of bias in individual studies [11]. The following risk of biases was analyzed: (1) selection bias, (2) performance bias, (3) detection bias, (4) attrition bias, and (5) reporting bias. 


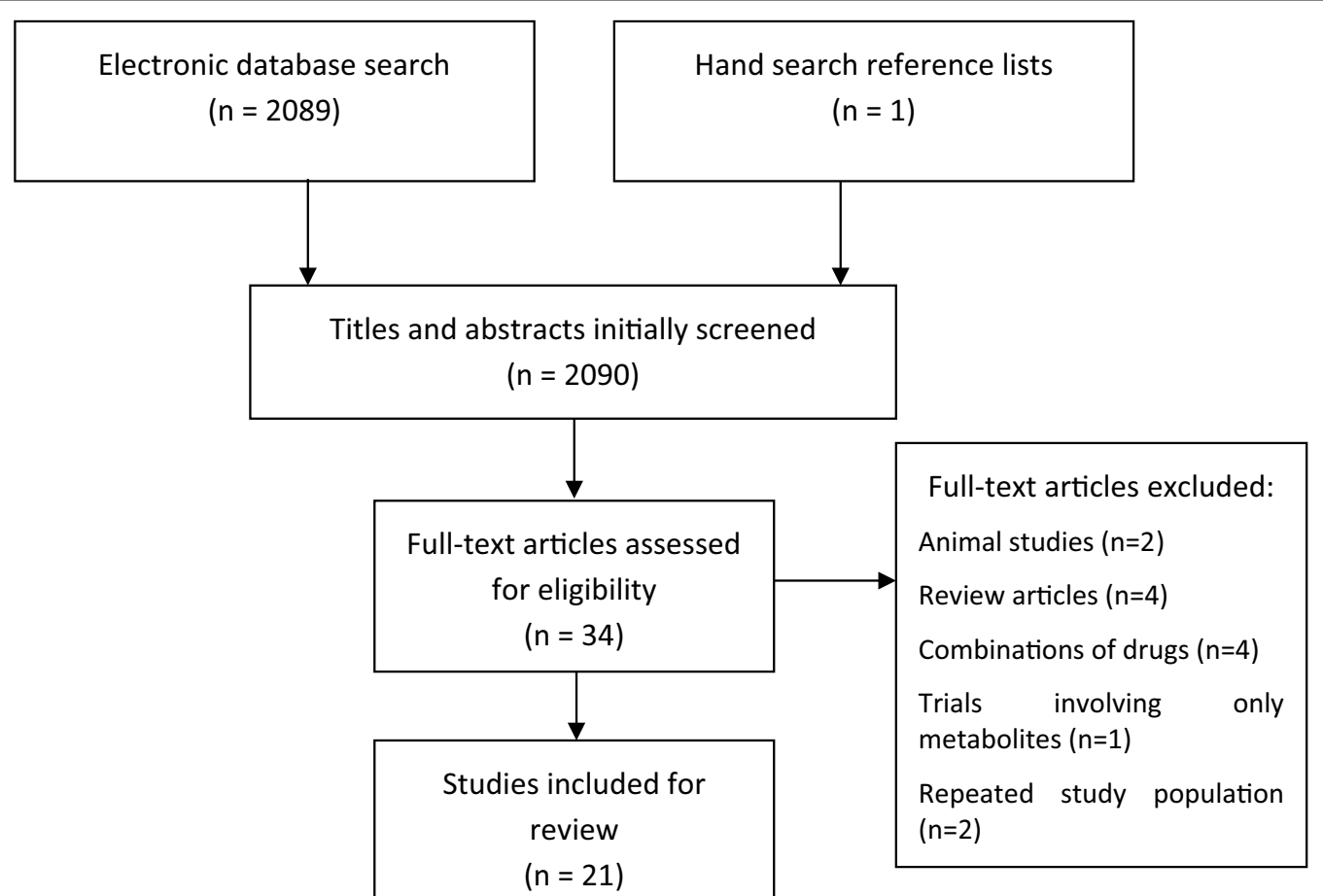

Fig. 1 PRISMA flow diagram of the study selection process

\section{Results}

Twenty-one articles were included in this review: seven are about fluvoxamine (FLV) [18-24], two about fluoxetine (FLX) [12, 13], sertraline [14, 15], venlafaxine (VEN) $[25,26]$, duloxetine $[27,28]$ and mirtazapine [30, 31], one about escitalopram [16], citalopram [17], trazodone [29] and bupropion [32]. No studies were found about paroxetine, milnacipran or agomelatine.

Eight studies were from Sweden, six were from Japan, four from Germany, two were from the United States of America (USA), and one from France and Switzerland. The studies reviewed included 2375 participants of which 733 were smokers. In terms of gender distribution, the vast majority of the studies reviewed recruited more female participants (64.31\%) than male participants $(35.69 \%)$. The average age of the subjects included in the studies is 45.53 years. A summary of results is given in Table 1 and the risk of bias in individual studies based on Cochrane Collaboration's tool for assessing risk of bias is given in Table 2. As shown in Table 2, selection and reporting bias are the most frequent, with seven of twenty-one studies assessed with high risk for selection bias and four with high risk of reporting bias. No detection and attrition bias were found, although the risk of bias was not always clear.

\section{Discussion}

As mentioned above, tobacco interferes with drug metabolism essentially by the action of PAH that have effects through the induction of CYP 1A1, CYP1A2 and CYP2E1 [9].

All the antidepressants evaluated are metabolized in the liver by different types of cytochromes. With respect to SSRIs, citalopram is metabolized by CYP 2C19 and $3 \mathrm{~A} 4$ [33], fluoxetine by 2D6, 3A4 and 2C9 [33], fluvoxamine by $1 \mathrm{~A} 2$ and 2D6 [33], escitalopram by $2 \mathrm{C} 19,2 \mathrm{D} 6$ and 3A4 [16], and sertraline by 2D6, 3A4, 2C9 and 2C19 [33]. Regarding SNRI, venlafaxine is metabolized by CYP 2D6, $3 \mathrm{~A} 4$ and $2 \mathrm{C} 9$ [33], and duloxetine by 2D6 and 1A2 [27]. Trazodone is metabolized by CYP 2D6 [29], mirtazapine by $1 \mathrm{~A} 2,2 \mathrm{D} 6$ and $3 \mathrm{~A} 4$ [31], and bupropion by $2 \mathrm{~B} 6$ [32] .

SSRI, except fluvoxamine, have little research on the effects of tobacco consumption in their serum levels. The trials performed with sertraline, escitalopram and citalopram showed no influence of smoking on their pharmacokinetics. However, the study on citalopram was based on a population in a restricted age group (all subjects were younger than 21 years) and studies regarding sertraline and escitalopram were not randomized and had many important limitations like the possibility of interactions with other drugs. The fluoxetine concentrations 


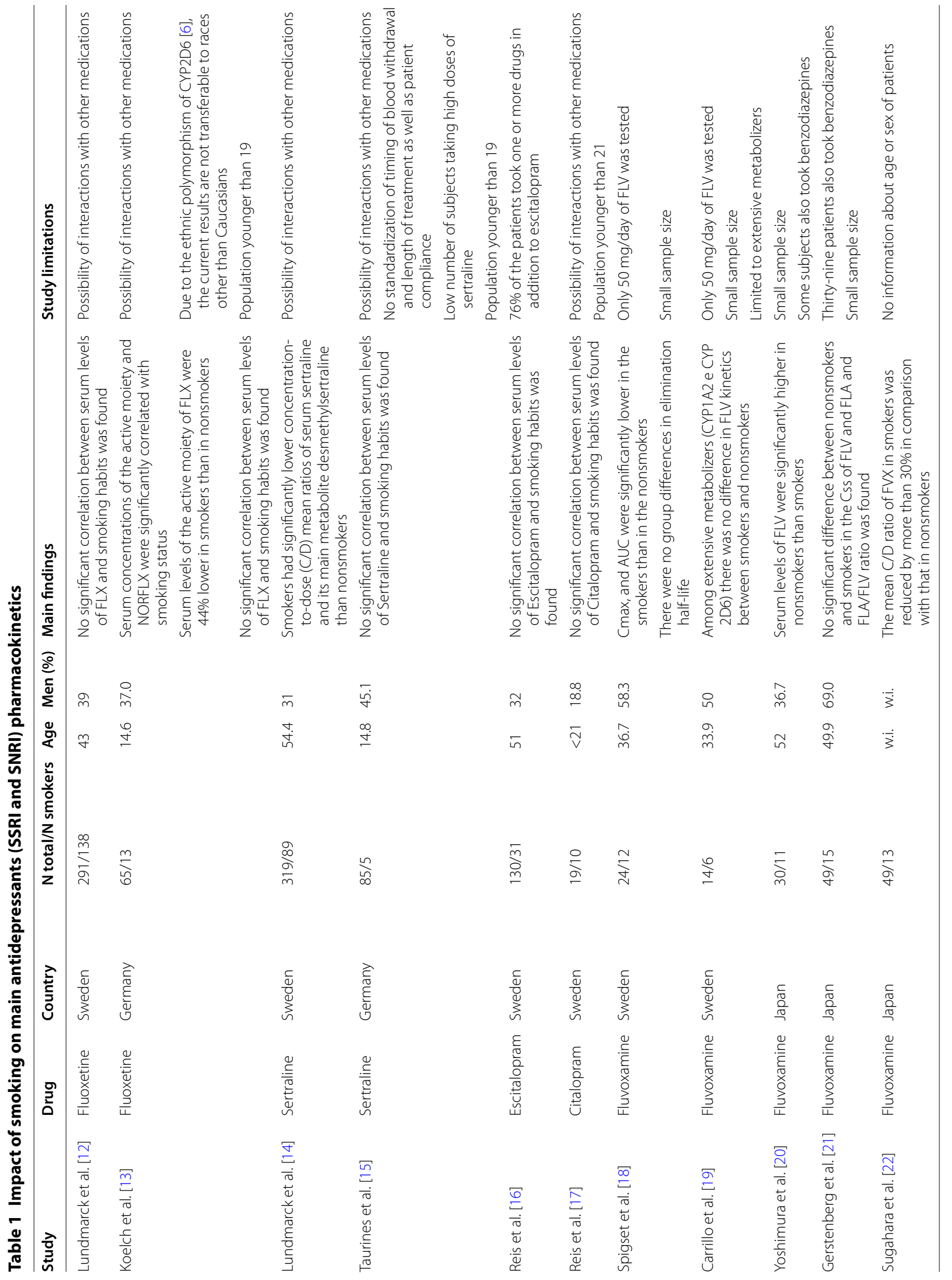




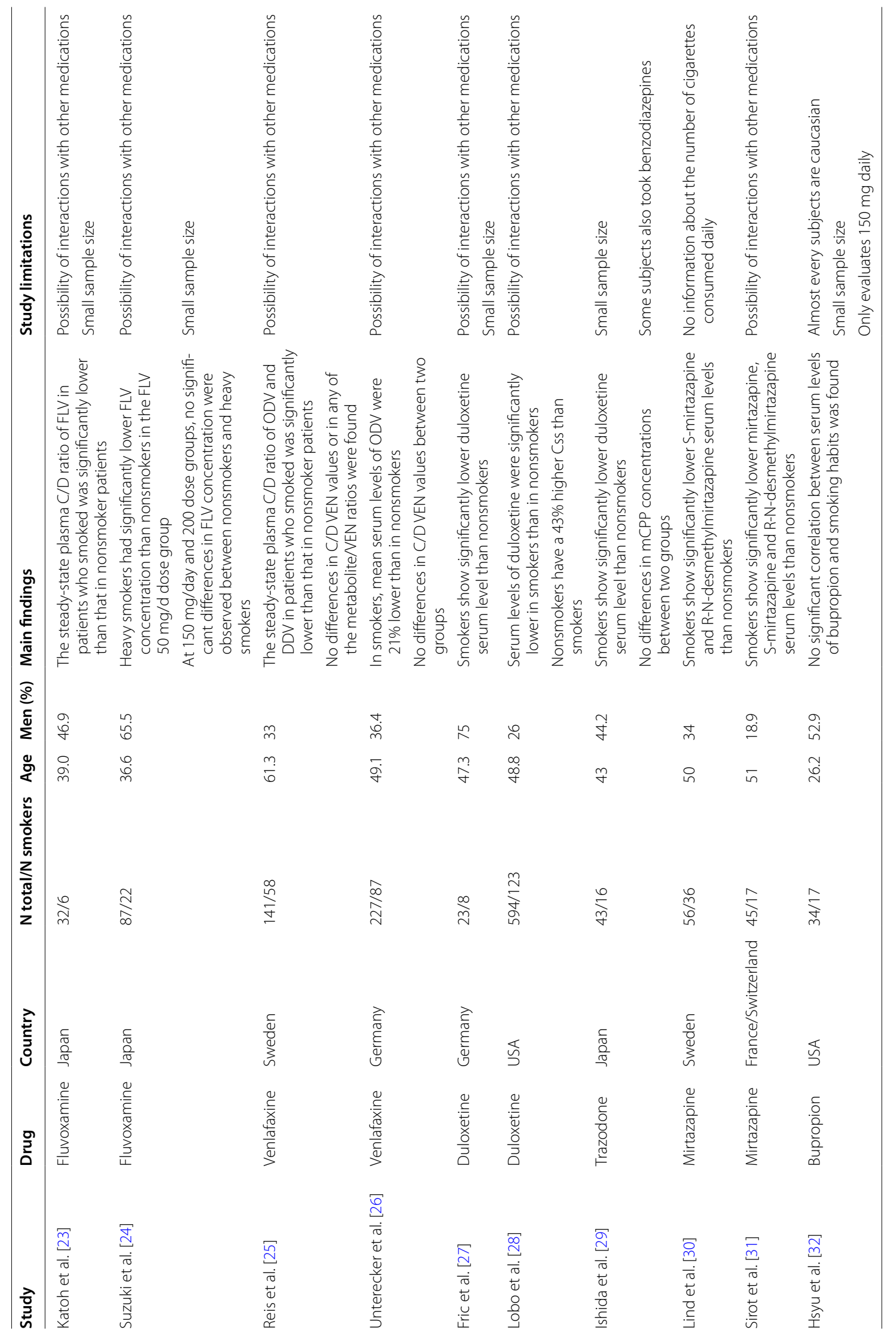


Table 2 Assessment of risk of bias in individual studies

\begin{tabular}{llllll}
\hline Study & Selection bias & Performance bias & Detection bias & Attrition bias & Reporting bias \\
\hline Lundmarck et al. [12] & - & - & - & $?$ & - \\
Koelch et al. [13] & $?$ & $?$ & - & - & + \\
Lundmarck et al. [14] & - & - & - & - & + \\
Taurines et al. [15] & $?$ & $?$ & - & - & $?$ \\
Reis et al. [16] & $?$ & $?$ & - & $?$ & + \\
Reis et al. [17] & + & $?$ & - & - & $?$ \\
Spigset et al. [18] & - & - & - & - & - \\
Carrillo et al. [19] & - & - & - & - & $?$ \\
Yoshimura et al. [20] & - & + & - & $?$ & $?$ \\
Gerstenberg et al. [21] & - & - & - & - & $?$ \\
Sugahara et al. [22] & $?$ & $?$ & - & - & $?$ \\
Katoh et al. [23] & + & - & - & - & $?$ \\
Suzuki et al. [24] & + & - & - & $?$ \\
Reis et al. [25] & + & $?$ & - & $?$ \\
Unterecker et al. [26] & - & - & - & $?$ \\
Fric et al. [27] & + & - & - & - & - \\
Lobo et al. [28] & - & - & - & - & - \\
Ishida et al. [29] & $?$ & - & - & - & - \\
Lind et al. [30] & + & - & - & - \\
Sirot et al. [31] & + & - & - & - \\
Hsyu et al. [32] & + & - & - & -
\end{tabular}

+ high risk of bias, - low risk of bias, ? unclear risk of bias

did not differ between the two study groups but the levels of its active metabolite, norfluoxetine, were higher in the group of smokers. Since norfluoxetine is an active metabolite, such association may impact the response of patients to take fluoxetine as well as implications with increased half-life of this molecule, which is already long, for example, by drug bioaccumulation and the ability to induce serotonin syndromes. Fluvoxamine has the greater evidence of decreased serum levels when associated with tobacco consumption. Although not proved, most studies suggest that there may be an association between the biotransformation of fluvoxamine and the activity of CYP1A2. It could not be excluded, however, that other factors may account for such a difference. Reasons include a possible association between smoking and fluvoxamine absorption, as well as between smoking and the elimination of fluvoxamine by other metabolic pathways. Further studies are, therefore, needed to clarify the role of CYP1A2 and other specific CYP in fluvoxamine metabolism and to elucidate which of the various metabolic steps could be dependent on CYP1A2. The recommended therapeutic reference range of fluvoxamine is $60-230 \mathrm{ng} / \mathrm{mL}$ [5]. Several studies have shown relations between plasma concentrations and clinical effects [5]. As a possible bias of these studies we highlight that most data involves Japanese individuals. This may influence the effect size, given, that this population presents quantitative differences, sometimes substantial, the various cytochrome P450 enzymes.

Assays for the SNRIs provide more consistent results than those involving SSRIs. Research on venlafaxine and duloxetine has similar designs, making result comparison easier and strengthening results. In both studies with venlafaxine, serum levels of both study groups showed no significant differences. Both pointed to a significant decrease in ODV levels. However, the pharmacodynamical effects of this are not completely understood, though many studies point to considerably weaker inhibition of serotonin and noradrenaline reuptake pumps when compared with venlafaxine itself [34]. For duloxetine, available data suggest a decrease in serum concentrations caused by tobacco consumption. In this drug, the available evidence is strong, based on multicenter randomized trials, involving a broad number of evaluated subjects. The effects of smoking status on duloxetine bioavailability can be attributed to the mechanism of duloxetine metabolism primarily by CYP1A2 enzyme. Smoking increases the expression of CYP1A2, which may explain the lower duloxetine bioavailability noted in smokers. The recommended therapeutic reference range of duloxetine is $30-120 \mathrm{ng} / \mathrm{mL}$ [5]. So far there is only a single retrospective analysis on plasma concentrations and 
clinical effects that has shown concentration-dependent improvement [5].

Data suggest a decrease in serum concentrations of trazodone caused by tobacco consumption and no influence in serum concentrations of trazodone's active metabolite $\mathrm{m}$-chloro-phenylpiperazine (mCPP). This reduction could be due to the enhancement of hydroxylation and N-oxidation of trazodone caused by PAH from cigarette smoke [29]. A concentration-response relationship for trazodone has not been established [29]; however, one study has suggested the presence of a linear relationship [35].

The two studies that evaluated the effect of smoking on mirtazapine's pharmacokinetics show significantly lower mirtazapine and their main active metabolites (S-mirtazapine and R-N-desmethylmirtazapine) serum levels in smokers than in nonsmokers. In vitro tomography study has shown that CYP1A2 is involved in 8-hydroxylation and possibly N-oxidation of mirtazapine [30]. Additionally, uridine diphosphate glucuronosyltransferases, another enzyme involved in the metabolism of mirtazapine, are inducible by smoking [30]. The recommended therapeutic reference range of mirtazapine is $30-80 \mathrm{ng} /$ $\mathrm{mL}$ [5]. In a study on patients with depression, responders to mirtazapine treatment presented higher plasma concentrations than non-responders [36].

In the study with bupropion serum levels, both study groups showed no significant differences [32]. This study only evaluated daily doses of $150 \mathrm{mg}$ of bupropion, reporting nothing on higher doses of drug.

Most antidepressants adverse effects are dose dependent, and some arise only when serum antidepressant levels reach a certain value [5]. Given that inhibition of CYP1A2 by tobacco smoke may decrease serum levels of some drugs, smoking cessation in heavy smokers taking such medication might lead to increased serum levels. Such an increase may cause adverse effects hitherto absent.

As mentioned above, the most frequent bias found was related with selection bias, which can lead to an over/ underestimation of the obtained results. Increasing the sample size and the use of control groups are recommended strategies to decrease this risk in future studies.

\section{Conclusions}

Despite numerous limitations in most studies, available evidence indicates a reduction in the concentration of serum levels of fluvoxamine, duloxetine, trazodone and mirtazapine in smoking patients when compared to nonsmokers. These differences raise the possibility of a semi-directed choice in antidepressant treatments, adapting the dose of these drugs and being aware of possible appearances of side effects after smoking cessation.
A personalized pharmacological treatment of depression could be made possible in a nearby future, guided by increasingly common and less expensive genotyping tools. For now, treatment personalization could be based on identifying phenotypes or external variables that influence antidepressant response or side effects. Further research is needed to improve our knowledge on the influence of smoking in depression pharmacological treatment.

\section{Authors' contributions}

$\mathrm{PO}, J \mathrm{R}, \mathrm{HD}$ and $\mathrm{NM}$ conceived the study and participated in its design and coordination. PO drafted the manuscript. NM, HD and JR revised the manuscript. PO, JR and NM revised it critically for important intellectual content. All authors read and approved the final manuscript.

\section{Author details}

${ }^{1}$ Psychiatry Department, Coimbra Hospital University Centre, Praceta Mota Pinto, 3000-075 Coimbra, Portugal. ${ }^{2}$ Documentation Department, Coimbra Hospital University Centre, Coimbra, Portugal.

\section{Acknowledgements}

We would like to thank the anonymous peer reviewers for their insightful comments on this paper.

\section{Competing interests}

The authors declare that they have no competing interests.

\section{Availability of data and materials}

The datasets used and/or analyzed during the current study available from the corresponding author on reasonable request.

Received: 30 November 2016 Accepted: 24 February 2017

Published online: 06 March 2017

\section{References}

1. Goff DC, Henderson DC, Amico E. Cigarette smoking in schizophrenia: relationship to psychopathology and medication side effects. Am J Psychiatry. 1992;149(9):1189-94.

2. Winterer G. Why do patients with schizophrenia smoke? Curr Opin Psychiatry. 2010;23:112-9.

3. Bromet E, Andrade LH, Hwang I, Sampson NA, Alonso J, de Girolamo G, et al. Cross-national epidemiology of DSM-IV major depressive episode. BMC Med. 2011;9:90.

4. Bastos H, Polido F, Saraiva C. História da Psiquiatria. In: Saraiva C, Cerejeira J, editors. Psiquiatria fundamental. Coimbra: Lidel Edições Técnicas; 2014.

5. Haji EO, Hiemke C, Pfuhlmann B. Therapeutic drug monitoring for antidepressant drug treatment. Curr Pharm Des. 2012;18(36):5818-27.

6. Ma Q, Lu AY. Pharmacogenetics, pharmacogenomics, and individualized medicine. Pharmacol Rev. 2011;63:437-59.

7. Hiemke C, Baumann P, Bergemann N, Conca A, Dietmaier O, Egberts K, et al. AGNP consensus guidelines for therapeutic drug monitoring in psychiatry: update 2011. Pharmacopsychiatry. 2011;44(6):195-235.

8. Kim YH, Bae YJ, Kim HS, Cha HJ, Yun JS, Shin JS, et al. Measurement of human cytochrome P450 enzyme induction based on mesalazine and mosapride citrate treatments using a luminescent assay. Biomol Ther. 2015;23(5):486-92.

9. Zevin S, Benowitz N. Drug interactions with tobacco smoking: an update. Clin Pharmacokinet. 1999;36(6):425-38.

10. Moher D, Liberati A, Tetzlaff J, Altman DG. The PRISMA Group: preferred reporting items for systematic reviews and meta-analyses: the PRISMA statement. Open Med. 2009;3:123-30.

11. Higgins J, Green S. Cochrane handbook for systematic reviews of interventions Version 5.1.0. The Cochrane Collaboration. 2011. http://www. cochrane-handbook.org. Accessed June 2016. 
12. Lundmark J, Margareta R, Finn B. Serum concentrations of fluoxetine in the clinical treatment setting. Ther Drug Monit. 2001;23:139-47.

13. Koelch M, Pfalzer A, Kliegl K, Rothenhöfer S, Ludolph AG, Fegert JM, et al. Therapeutic drug monitoring of children and adolescents treated with fluoxetine. Pharmacopsychiatry. 2012;45:72-6.

14. Lundmark J, Margareta R, Finn B. Therapeutic drug monitoring of sertraline: variability factors as displayed in a clinical setting. Ther Drug Monit. 2000;22:446-54.

15. Taurines R, Burger R, Wewetzer C, Pfuhlmann B, Mehler-Wex C, Gerlach $M$, et al. The relation between dosage, serum concentrations, and clinical outcome in children and adolescents treated with sertraline: a naturalistic study. Ther Drug Monit. 2013;35:84-91.

16. Reis M, Cherma M, Carlsson B, Bengtsson F. Therapeutic drug monitoring of escitalopram in an outpatient setting. Ther Drug Monit. 2007;29:758-66.

17. Reis M, Olson G, Carlsson B, Bengtsson F. Serum levels of citalopram and its main metabolites in adolescent patients treated in a naturalistic clinical setting. J Clin Psychopharmacol. 2002;22:406-13.

18. Spigset O, Carleborg L, Hedenmalm K, Dahlqvist R. Effect of cigarette smoking on fluvoxamine pharmacokinetics in humans. Clin Pharmacol Ther. 1995;4:399-403.

19. Carrillo J, Dahl M, Svensson J, Alm C, Rodriguez I, Bertilsson L. Disposition of fluvoxamine in humans is determined by the polymorphic CYP2D6 and also by the CYP1A2 activity. Clin Pharmacol Ther. 1996:2:183-90.

20. Yoshimura R, Ueda N, Jun Nakamura J, Eto S, Matsushita M. Interaction between fluvoxamine and cotinine or caffeine. Neuropsychobiology. 2002:45:32-5

21. Gerstenberg G, Aoshima T, Fukasawa T, Yoshida K, Takahashi H, Higuchi $\mathrm{H}$, et al. Effects of the CYP $2 \mathrm{~d} 6$ genotype and cigarette smoking on the steady-state plasma concentrations of fluvoxamine and its major metabolite fluvoxamino acid in Japanese depressed patients. Ther Drug Monit. 2003;25:463-8

22. Sugahara $\mathrm{H}$, Maebara $\mathrm{C}$, Ohtani $\mathrm{H}$, Handa M, Ando K, Mine K, et al. Effect of smoking and CYP2D6 polymorphisms on the extent of fluvoxaminealprazolam interaction in patients with psychosomatic disease. Eur J Clin Pharmacol. 2009;65:699-704.

23. Katoh Y, Uchida S, Kawai M, Takei N, Mori N, Kawakami J, et al. Effects of cigarette smoking and cytochrome p450 $2 \mathrm{~d} 6$ genotype on fluvoxamine concentration in plasma of Japanese patients. Biol Pharm Bull. 2010:33:285-8.

24. Suzuki Y, Sugai T, Fukui N, Watanabe J, Ono S, Inoue Y, et al. CYP2D6 genotype and smoking influence fluvoxamine steady-state concentration in Japanese psychiatric patients: lessons for genotype-phenotype association study design in translational pharmacogenetics. J Psychopharmacol. 2011;25:908-14.
25. Reis $M$, Lundmark J, Bjork H, Bengtsson F. therapeutic drug monitoring of racemic venlafaxine and its main metabolites in an everyday clinical setting. Ther Drug Monit. 2002;24:545-53.

26. Unterecker S, Hiemke C, Greiner C, Haen E, Jabs B, Deckert J, et al. The effect of age, sex, smoking and co-medication on serum levels of venlafaxine and o-desmethylvenlafaxine under naturalistic conditions. Pharmacopsychiatry. 2012:45:229-35.

27. Fric M, Pfuhlmann B, Laux G, Riederer P, Distler G, Artmann S, et al. The influence of smoking on the serum level of duloxetine. Pharmacopsychiatry. 2008:41:151-5.

28. Lobo E, Quinlan T, O'Brien L, Knadler MP, Heathman M. Population pharmacokinetics of orally administered duloxetine in patients: implications for dosing recommendation. Clin Pharmacokinet. 2009;48:189-97.

29. Ishida M, Otani K, Kaneko S, Ohkubo T, Osanai T, Yasui N, Mihara K, Higuchi $\mathrm{H}$, Sugawara K. Effects of various factors on steady state plasma concentrations of trazodone and its active metabolite m-chlorophenylpiperazine. Int Clin Psychopharmacol. 1995;10(3):143-6.

30. Lind AB, Reis M, Bengtsson F, Jonzier-Perey M, Powell Golay K, Ahlner $\mathrm{J}$, et al. Steady-state concentrations of mirtazapine, N-desmethylmirtazapine, 8-hydroxymirtazapine and their enantiomers in relation to cytochrome P450 2D6 genotype, age and smoking behaviour. Clin Pharmacokinet. 2009:48(1):63-70

31. Sirot EJ, Harenberg S, Vandel P, Lima CA, Perrenoud P, Kemmerling K, et al. Multicenter study on the clinical effectiveness, pharmacokinetics, and pharmacogenetics of mirtazapine in depression. J Clin Psychopharmacol. 2012;32(5):622-9.

32. Hsyu PH, Singh A, Giargiari TD, Dunn JA, Ascher JA, Johnston JA. Pharmacokinetics of bupropion and its metabolites in cigarette smokers versus nonsmokers. J Clin Pharmacol. 1997:37(8):737-43.

33. Sandson NB. Drug interactions casebook: the cytochrome P450 system and beyond. Chicago: American Psychiatric Publishing; 2003.

34. Muth E, Moyer J, Haskins J, Andree TH, Husbands GM. Biochemical, neurophysiological, and behavioral effects of wy-45,233 and other identified metabolites of the antidepressant venlafaxine. Drug Dev Res. 1991;23:191-9

35. Monteleone P, Gnocchi G, Delrio G. Plasma trazodone concentrations and clinical response in elderly depressed patients: a preliminary study. J Clin Psychopharmacol. 1989;9(4):284-7.

36. Grasmäder K, Verwohlt PL, Kühn KU, Frahnert C, Hiemke C, Dragicevic $A$, et al. Relationship between mirtazapine dose, plasma concentration, response, and side effects in clinical practice. Pharmacopsychiatry. 2005:38(3):113-7.

\section{Submit your next manuscript to BioMed Central and we will help you at every step:}

- We accept pre-submission inquiries

- Our selector tool helps you to find the most relevant journal

- We provide round the clock customer support

- Convenient online submission

- Thorough peer review

- Inclusion in PubMed and all major indexing services

- Maximum visibility for your research

Submit your manuscript at www.biomedcentral.com/submit
BioMed Central 